\title{
Frontières
}

\section{Entre transfert et dépendance}

\section{Les peurs du déprimé et les aléas du traitement}

\section{Marc-Alain Wolf et Yaël Wolf}

Volume 21, numéro 2, printemps 2009

Détresse psychique et antidépresseurs

URI : https://id.erudit.org/iderudit/039461ar

DOI : https://doi.org/10.7202/039461ar

Aller au sommaire du numéro

Éditeur(s)

Université du Québec à Montréal

ISSN

1180-3479 (imprimé)

1916-0976 (numérique)

Découvrir la revue

Citer cet article

Wolf, M.-A. \& Wolf, Y. (2009). Entre transfert et dépendance : les peurs du déprimé et les aléas du traitement. Frontières, 21(2), 73-76.

https://doi.org/10.7202/039461ar d'utilisation que vous pouvez consulter en ligne.

https://apropos.erudit.org/fr/usagers/politique-dutilisation/ 


\section{ENTRE TRANSFERT ET DÉPENDANCE Les peurs du déprimé et les aléas du traitement}

Marc-Alain Wolf, M.D., Ph. D., CSPQ (Psy), psychiatre, Institut universitaire Douglas, professeur, Département de psychiatrie, Université McGill, Département de psychologie, UQAM.

Yaël Wolf, M.D., C.M., résidente en psychiatrie, Faculté de médecine, Université McGill.

L'antidépresseur reste un agent thérapeutique controversé dont l'utilisation croissante n'est pas toujours bien encadrée par la science. Des facteurs économiques et idéologiques influencent, entre autres, le très lucratif marché des psychotropes. Dans ce texte, après avoir décrit les principaux symptômes cliniques de la dépression, nous soulignerons les limites des classifications actuelles qui ne permettent pas toujours au praticien de prédire le type de traitement qui sera le plus efficace. Nous exprimerons ensuite notre point de vue sur le domaine, si vaste, des psychothérapies en évoquant notamment les principes communs aux différentes techniques et les choix ou préférences épistémologiques qui s'offrent au clinicien et au patient.

\section{LA DÉPRESSION : PRÉSENTATION CLINIQUE ET CLASSIFICATION}

La dépression est une maladie contemporaine, expansive mais encore floue. Dans leur "guide de psychothérapie interpersonnelle », Weissman et al. (2006) caractérisent la dépression par une humeur dysphorique ou une perte d'intérêt ou de plaisir dans la plupart des activités et loisirs habituels. La dysphorie est prédominante, persistante et accompagnée d'autres symptômes (troubles de l'appétit, perte ou gain de poids, troubles du sommeil, agitation ou ralentissement psychomoteur, baisse d'énergie, sentiments d'inutilité ou de culpabilité, difficulté à se concentrer, à penser ou à prendre des décisions, pensées de mort ou de suicide, tentatives de suicide). Ces auteurs considèrent qu'il s'agit d'un trouble à multiples facettes, mais l'intérêt clinique de la division en sous-types (endogène, mélancolique, saisonnière, etc.) ne fait pas l'unanimité. Dans leur souci d'objectivité statistique, les auteurs du DSM-IV (Association américaine de psychiatrie, 1996) décrivent neuf critères de dépression et exigent, pour porter le diagnostic d'épisode dépressif majeur, la présence de cinq au moins de ces critères pendant une période continue de deux semaines. En outre, le tableau clinique doit représenter un changement par rapport au fonctionnement antérieur et un des symptômes présents doit être obligatoirement une humeur dépressive ou une perte d'intérêt ou de plaisir. Dans leur manuel de psychiatrie dont la première édition remonte à 1960, Henri Ey et al. (1978) reconnaissent à leur tour que le problème de la classification des états dépressifs est difficile. Si, classiquement, on distingue bien les dépressions endogènes (ou mélancoliques) des dépressions névrotiques et réactionnelles, on n'a jamais cessé en fait de discuter et des formes de passage et de l'étiologie (endogène ou exogène), et de la différence de pronostic ou des indications thérapeutiques. L'apparition des premiers antidépresseurs au tournant des années 1950 et 1960 et du lithium au début des années 1970 a entraîné une remise en question des anciennes classi- fications. La question centrale était (et reste aujourd'hui) de déterminer quels types de dépression bénéficient du traitement pharmacologique. Longtemps les psychiatres se sont entendus pour opposer les dépressions endogènes ou mélancoliques, d'étiologie organique et nécessitant des traitements biologiques (antidépresseurs ou électrochocs) aux dépressions névrotico-réactionnelles relevant principalement d'un déterminisme psychologique ou évènementiel et justifiant le recours à des traitements psychothérapeutiques. Cette distinction utile et respectable a été battue en brèche par les promoteurs d'une psychiatrie scientifique qui la jugeaient non fiable mais aussi par certains psychiatres psychanalystes témoignant humblement de leur surprise (stupéfaction?) lorsque certains de leurs patients en analyse depuis des années, souffrant apparemment d'une forme névrotique et multi-récidivante de dépression, répondaient miraculeusement au traitement antidépresseur ou thymorégulateur. L'approche athéorique et quantitative de la dépression par les différentes éditions du DSM a fini par ruiner, en tout cas en médecine, le dualisme des anciens et l'espoir de limiter l'usage de la pharmacothérapie dans le domaine de plus en plus vaste de la dépression. Le même phénomène de conquête (et de procès) s'est opéré dans le champ clinique voisin de l'anxiété. La démonstration de l'efficacité de certains antidépresseurs dans un nombre croissant de troubles anxieux a conduit à une remise en cause de la légitimité de la psychanalyse (aux effets cliniques incertains) dans ce qui était jusque-là son domaine réservé. 


\section{PSYCHOTHÉRAPIES:}

\section{DIVERSITÉ ET INTÉGRATION}

Le domaine de la psychothérapie est lui aussi en expansion continue. Il existe à ce jour plusieurs centaines de variétés de thérapie. Les plus classiques dérivent des quatre approches fondamentales suivantes: la psychanalyse, l'approche objective-descriptive (dont relèvent les thérapies comportementales et cognitives), l'approche existentielle et les thérapies interpersonnelles. Si les oppositions idéologiques et les rivalités d'écoles ont longtemps retardé le dialogue entre thérapeutes d'obédiences diverses, il existe maintenant un mouvement original de rapprochement, d'échanges et d'intégration. Donnons-en ici, rapidement, quelques repères (voir Goldfried et al., 1998). French est l'un des premiers à comparer la psychanalyse et le conditionnement pavlovien, observant par exemple des similitudes entre répression et extinction. Alexander propose une compréhension de la thérapie psychanalytique à partir des concepts de la théorie de l'apprentissage. Watson propose d'appliquer aux thérapeutes la distinction si utile avec les patients entre ce qui est dit et ce qui est fait. Carl Rogers invite les praticiens à se libérer des contraintes de la théorie et à observer aussi directement que possible ce qui se passe vraiment dans une psychothérapie. Les caractéristiques personnelles du thérapeute contribueraient davantage au processus de changement que son école de pensée.

Certaines recherches visent à identifier des principes de changement communs aux différentes thérapies qui rendraient compte de leur efficacité. Les facteurs spécifiques qui permettent aux différentes écoles d'exister et qui reçoivent d'elles une grande attention seraient paradoxalement de moindre importance. Parmi les facteurs communs, citons l'existence d'un cadre thérapeutique bien défini, l'identification d'objectifs de changement, l'importance de la relation interpersonnelle et de son pouvoir d'influence, l'engagement des interlocuteurs dans le processus, etc.

Les diverses approches thérapeutiques s'adressent prioritairement à des aspects différents du fonctionnement psychologique comme l'émotion, l'inconscient, la pensée consciente et le comportement. Certains auteurs comme Ryle (1990) adoptent un modèle psychopathologique séquentiel et circulaire: les fonctions psychologiques interagissent l'une sur l'autre et toute intervention, quel que soit son point d'impact, exercera une influence générale sur l'ensemble du cycle.

Mais il faut reconnaître aussi au départ des «représentations du monde» opposées: là où le comportementalisme, par

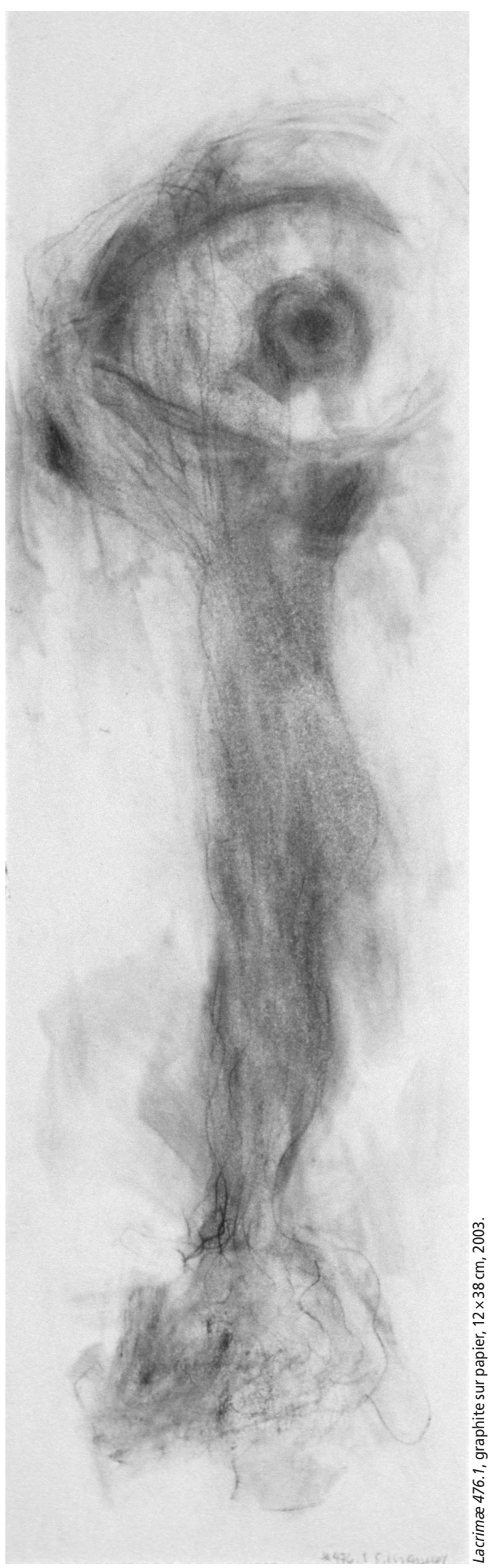

exemple, prône le réalisme (existence d'un monde commun à tous) et l'«extraspection» (recherche des mobiles du comportement à l'extérieur de l'individu), la psychanalyse privilégie l'idéalisme (le monde comme construction de notre pensée), la subjectivité (le monde comme cadre de référence particulier à chacun) et l'introspection (recherche des motivations du comportement à l'intérieur du sujet). Messer (1998), s'inspirant des travaux du critique littéraire Northrop Frye, propose une formulation plus littéraire de cette opposition: l'approche comportementale procéderait d'une vision «comique » de la réalité, qui cherche à atteindre le bonheur en déplaçant (ou en faisant disparaître) les difficultés psychologiques et les obstacles de l'environnement. La psychanalyse, de son côté, développerait une vision plus «tragique» en considérant la souffrance et les conflits comme des expériences humaines inévitables qu'il faut accepter, comprendre, assumer et éventuellement dépasser.

Ces divergences épistémologiques ne représentent une barrière à l'intégration qu'à un niveau d'abstraction théorique et non à un palier intermédiaire comme les stratégies cliniques. Dans l'exercice quotidien de la psychothérapie, les approches psychodynamiques et comportementales peuvent être utilisées de manière complémentaire: les premières favorisent la prise de conscience, la compréhension du problème, les secondes mettent en œuvre et contrôlent le processus de changement; les unes peuvent réussir là où les autres échouent.

Le choix d'une approche peut-il dépendre des «préférences épistémologiques» du clinicien? Royce et Mos (1980) ont évalué le «profil psycho-épistémologique» d'un groupe de thérapeutes en distinguant les trois axes suivants: rationalisme, empirisme et «métaphorisme», ce dernier désignant un type de pensée qui privilégie le raisonnement analogique et l'élaboration de sens à l'observation en elle-même. Comme on pouvait s'y attendre, psychanalystes et comportementalistes ont présenté des profils contrastés: alors que les premiers adhéraient massivement au métaphorisme, les seconds partageaient plus équitablement leurs préférences. Ces derniers seraient plus ouverts au mouvement intégratif en raison d'une plus grande "flexibilité épistémologique ».

Le transfert est-il une modalité commune à toutes les formes de psychothérapie ? Si la psychanalyse a bien décrit le lien névrotique qui finit par attacher le client à son analyste, il est vraisemblable que ce phénomène se développe dans toute forme d'interaction asymétrique, de relation d'aide ou d'influence prolongée et approfondie, au cours de laquelle le thérapeute, le maître ou le gourou finit par occuper une position ambiguë qui suscite chez l'interlocuteur toute une série d'identifications et de projections. Le colloque singulier devient alors un jeu de chaises musicales dans lequel les participants adoptent des positions différentes, portent des masques variés et finissent par développer une relation interpersonnelle 
particulière, à vocation heuristique dont l'analyse pourra, le cas échéant, révéler la part prépondérante jouée, non par la personnalité réelle du maître (du gourou ou du thérapeute) mais par le vécu subjectif (présent et passé, réel et imaginaire, conscient et inconscient) du sujet.

L'analogie proposée ici entre toutes les formes de lien thérapeutique, mais aussi entre le dispositif psychothérapique et celui des sectes est sans doute abusive et injuste, mais elle permet d'illustrer les illusions et les peurs qui mobilisent les individus souffrants lorsque se pose à eux la question d'une aide à rechercher. Les travaux sur l'hypnose (cet ancêtre de la psychothérapie) ont montré jadis qu'il existait une fraction de la population qui répondait particulièrement bien à cette forme d'influence contrairement à un autre groupe qui résistait complètement à cette approche. Des facteurs caractériels nous prédisposent à rechercher ou à éviter une relation d'aide qui semble exiger un assujettissement ou une dépendance à autrui. L'engagement du client ou du patient dans la relation thérapeutique, si importante pour assurer son efficacité, dépend de la capacité de la personne à tolérer cet exercice d'humilité-humiliation qui consiste à reconnaître son impuissance, d'un côté, et à attribuer un pouvoir à autrui de l'autre. La multiplication des nouvelles Églises, des sectes, des techniques de croissance personnelle, des médecines alternatives, des écoles plus ou moins sérieuses de psychothérapie montre à quel point le marché de la dépendance est florissant. Pour soulager leurs inquiétudes et leurs craintes, leurs peines et leurs souffrances, pour remplir leur vide existentiel parfois aussi, nos contemporains sont capables d'une crédulité sans limite.

\section{L'ANTIDÉPRESSEUR: UN AGENT THÉRAPEUTIQUE CONTROVERSÉ}

Depuis plus de cinquante ans, un nouveau venu perturbe ce marché. Le psychotrope a vite fait d'imposer sa présence, l'efficacité de sa mise en marché et la simplicité de son utilisation. L'antidépresseur est le fer de lance d'une industrie psychopharmacologique florissante mais il reste un outil controversé dans le traitement des troubles anxiodépressifs. Le principal reproche qui lui est fait tient au paradoxe suivant: la plupart des personnes qui en consomment pourraient ne pas en avoir besoin, la majorité des patients qui auraient besoin de ce traitement n'y ont pas accès ou refusent de s'y soumettre. La psychopharmacologie reste aujourd'hui une science fragile. La découverte des antidépresseurs est largement le fruit du hasard (Landry, 2001). En 1954, Bloch et ses collaborateurs rapportent l'effet antidépresseur d'un antituberculeux, l'iproniazide. Cette molécule inhibe la monoamine-oxydase (ou MAO), l'enzyme qui catabolise certains neurotransmetteurs du cerveau. Il sera le précurseur d'une classe d'antidépresseurs, les inhibiteurs de la monoamine-oxydase (ou IMAO). Toxique pour le foie, l'iproniazide sera retiré du marché mais une nouvelle molécule, la phénelzine, conçue synthétiquement à partir de l'iproniazide, deviendra en 1959 le premier IMAO approuvé comme antidépresseur. Les agents tricycliques sont également découverts de manière fortuite. En 1958, le Suisse Kuhn décrit les propriétés antidépressives de l'imipramine, synthétisée à l'origine dans le but de produire un neuroleptique dérivé de la chlorpromazine. Ainsi, c'est en voulant produire par manipulation moléculaire un nouvel antituberculeux et un nouveau neuroleptique que les chimistes des compagnies pharmaceutiques synthétisent, sans jamais l'avoir voulu, les premiers antidépresseurs.

La psychopharmacologie clinique a connu, ces vingt dernières années, d'incontestables succès comme la découverte d'une nouvelle classe d'antidépresseurs mieux tolérés, les inhibiteurs sélectifs de la recapture de sérotonine (ou ISRS). Elle a aussi démontré l'efficacité de certains antidépresseurs dans le traitement d'un grand nombre de troubles anxieux (paniques anxieuses, anxiété généralisée, phobies, troubles obsessifs compulsifs etc.). Mais ces avancées n'ont pas permis d'établir complètement son statut scientifique. Elle reste tributaire d'une nosographie psychiatrique imprécise et fluctuante et continue d'utiliser des échelles d'évaluation subjectives. Aucun test biologique ne permet aujourd'hui d'affirmer un diagnostic de dépression ou l'efficacité réelle d'un antidépresseur. Malgré le raffinement apparent des études cliniques, le grand nombre de patients investigués, l'entrâ̂nement consciencieux des évaluateurs, la sophistication des outils statistiques utilisés, il reste hasardeux de tenir pour acquis la plupart des résultats des études publiées. La supériorité affichée d'un médicament sur son concurrent peut être l'effet d'un biais méthodologique pas toujours facile à détecter. Le développement récent des méta-analyses qui recensent, évaluent et réinterprètent les résultats de plusieurs dizaines d'études n'a pas permis de répondre à toutes les questions en suspens. Les compagnies pharmaceutiques restent les maîtres d'œuvre de la recherche et de la publication des études qui permettront ou non la commercialisation de leurs produits. Elles jouissent, par leur puissance financière, d'un quasi-monopole sur l'in- formation transmise aux médecins. Elles sont tenues à certaines règles de rigueur et de probité mais cherchent toujours, bien entendu, à favoriser leurs produits, ce qu'elles peuvent faire de multiples façons: en sélectionnant les populations investiguées, en choisissant le devis méthodologique qui correspond le mieux au profil anticipé de leurs molécules, en publiant une partie seulement de leurs résultats, en soignant, à leur manière, la publication finale de leurs études, en adoptant enfin une stratégie marketing soumise davantage à des impératifs économiques qu'au respect de la vérité. L’un des phénomènes les plus troublants du processus de mise en marché des psychotropes tient à la durée limitée des brevets consentis aux compagnies pharmaceutiques. Pendant dix ou quinze ans, le prescripteur (et de plus en plus le consommateur) sera bombardé d'informations vantant les mérites de tel médicament mais, dès l'expiration du brevet et l'apparition de produits génériques commercialisés par d'autres compagnies, la communication s'interrompt brutalement et un silence assourdissant remplace le bruit entretenu jusque-là autour de la fameuse molécule. L'attrait de la nouveauté, les phénomènes de mode et d'usure médiatique, les engouements artificiellement construits et déconstruits n'épargnent pas le marché des psychotropes. Tout est fait pour promouvoir les nouveaux médicaments au coût élevé et aux avantages pas toujours démontrés, au détriment des molécules anciennes moins chères et qui, pour certaines d'entre elles, ont fait leur preuve. Ce phénomène est préoccupant parce qu'il pervertit la concurrence qui reste, dans une économie libérale, le garant d'une certaine rationalité et d'un certain équilibre.

L'effet placebo demeure un défi de taille pour la médecine moderne et un obstacle dans sa marche en avant. Il représente, dans le cas des antidépresseurs, une partie non négligeable de l'effet thérapeutique, parfois plus importante que l'effet spécifique attribuable objectivement à la molécule. La méthodologie des études cliniques en double aveugle contre placebo permet de neutraliser cet effet "parasite» en recherche mais il en va tout autrement en clinique. Pour un sujet pris individuellement, il est impossible d'évaluer précisément l'importance relative des effets placebo et spécifique. Si ce sujet répond au traitement, il ne répond pas forcément à la pilule mais à un processus complexe, interpersonnel, où la personne du prescripteur et celle du client, le contexte de la rencontre, les caractéristiques de l'interaction jouent à des degrés divers parmi d'autres facteurs. La demande du 
patient, ses préjugés positifs ou négatifs envers l'antidépresseur, ses craintes et ses attentes sont souvent déterminantes dans l'acceptation, la fidélité et la réponse au traitement. La plupart des médecins et des psychiatres tiennent compte, quand cela est possible, des préférences du client ou de son entourage. Il vaut mieux prescrire une molécule connue, ayant bonne presse et qu'un proche a déjà expérimentée avec succès, qu'un produit inconnu ou stigmatisé (à tort ou à raison) par le milieu. Cela vaut aussi dans le cadre institutionnel des hôpitaux où le médecin doit éviter d'imposer des médicaments qui n'ont pas la confiance du personnel infirmier, lequel joue un rôle déterminant dans le "service après-vente» et l'interface psychotropepatient. Les croyances du médecin, ses doutes, la force ou la faiblesse de sa «foi » pour une molécule donnée, sa capacité à la transmettre au patient et à sa famille, sont d'autres ingrédients possibles de l'effet placebo.

Qu'est-ce qui pousse ou retient un patient de prendre un antidépresseur? Qu'est-ce qui motive son attirance ou sa répulsion? Il n'y a pas de réponse simple à cette question. L'expérience clinique, rejoignant la logique et le bon sens, montre que l'individu souffrant et conscient de son mal acceptera plus facilement l'idée d'un traitement. Ainsi le déprimé se pliera plus facilement aux ordonnances de son médecin que le maniaque et le névrosé recherchera plus souvent une aide (psycho- ou pharmacothérapique) que le psychotique. Mais au-delà de cette première observation, il faut aussi distinguer des profils différents entre sujets. Je risquerai la distinction entre patient, consommateur et réfractaire. Le «patient» est celui qui n'a pas d'idée préconçue sur l'utilisation de psychotropes. Il adopte le "rôle» de malade, reconnaît la compétence du médecin et lui délègue la responsabilité de décider pour lui. Il accepte sans trop discuter le traitement qui lui est proposé, comme il accepterait, le cas échéant, de ne pas en recevoir. Le «consommateur» prend les devants. Il exige d'emblée une prescription. Il est à la recherche d'une molécule qui le soulagera de son mal ou stimulera sa performance. Il a souvent l'habitude de recourir à certains moyens chimiques (tabac, alcool, stimulants divers, drogues, etc.) pour se rendre (croit-il) la vie plus facile. Il consomme l'antidépresseur ou le tranquillisant pour calmer ses angoisses existentielles, anesthésier sa peine ou sa rage, lever certaines inhibitions. Le "réfractaire» refuse d'emblée l'idée sacrilège de recourir à un traitement psychotrope qu'il considère comme un agent toxique ou comme une atteinte à sa liberté de sujet. L'idéologie écologique ou libertaire anime souvent ces individus militants. Le médicament est appréhendé comme un poison chimique ou un instrument de domination, de contrôle social et de déshumanisation. La peur de la dépendance au produit est aussi un élément de ce refus global. Les explications médicales resteront vaines. Pour ces militants, le médicament psychiatrique n'est qu'une drogue comme une autre.

\section{LES LOIS DU MARCHÉ ET LES PEURS DU DÉPRIMÉ}

Le mal de vivre dans nos sociétés, qu'il prenne ou non la forme d'une maladie répertoriée dans les manuels de psychiatrie, alimente une industrie de la relation d'aide en croissance ininterrompue. Si la psychanalyse ne suscite plus aujourd'hui le même engouement que par le passé, le champ de la psychothérapie se diversifie avec la multiplication de nouvelles écoles et de nouvelles techniques. Le nombre de prescriptions d'antidépresseurs s'envole, lui aussi, et le marché des psychotropes génère des profits impressionnants. La concurrence entre les deux formes de thérapie est inégale et tourne de plus en plus à l'avantage du médicament. Celui-ci est plus facile à administrer, son efficacité supposée est plus rapide, mieux établie scientifiquement et son coût est en grande partie pris en charge par l'État. Mais l'opposition entre les deux approches n'est plus aussi marquée que jadis. Le modèle bio-psycho-social de compréhension du trouble mental favorise aujourd'hui le rapprochement et la synergie entre l'intervention psychologique et pharmacologique. On ne demande plus au client de choisir entre la molécule et la psychothérapie, on lui offre les deux! Les récalcitrants auront le choix de refuser tout ou partie de cette offre. Le marché de la souffrance psychologique est soumis aux aléas de l'offre et de la demande. La mode est aux techniques orientales, aux communautés d'entraide, aux greffes ou aux chimères psycho-spirituelles ou psycho-religieuses. Malgré des efforts pour contrôler et évaluer les traitements offerts, il y a encore, aujourd'hui, des tendances lourdes qui déstabilisent ce marché, des pathologies de l'offre et de la demande qui découragent parfois tout effort de rationalisation. Citons parmi elles la prolifération anarchique de l'offre en psychothérapie, la crédulité sans limite de certains clients, le pouvoir excessif de l'industrie et du facteur économique dans la recherche et la clinique psychopharmacologiques, la confusion croissante entre le statut de patient et celui de consommateur.
C'est là tout le paradoxe de la situation. Les personnes les plus à risque de développer une relation de dépendance sont aussi celles qui n'hésitent pas à consulter et à chercher de l'aide. Les plus méfiantes, celles qui sont le mieux préparées à résister aux promesses des lendemains qui chantent, se replient sur elles-mêmes, s'isolent dans leur refus parfois hautain de s'ouvrir à autrui et de reconnaître leurs souffrances, leurs impasses, leur impuissance.

\section{Bibliographie}

ASSOCIATION AMÉRICAINE DE PSYCHIATRIE (1996). DSM-IV, Manuel diagnostique et statistique des troubles mentaux, traduction française, Paris, Masson.

EY, H., P. BERNARD et C. BRISSET (1978). Manuel de psychiatrie, $5^{\mathrm{e}}$ édition, Paris, Masson.

GOLDFRIED, M.R. et C.F. NEWMAN (1998). "Histoire de l'intégration », dans J.C. NORCROSS et M.R. GOLDFRIED (dir.), Psychothérapie intégrative, Paris, Desclée de Brouwer.

LANDRY, P. (2001) "Antidépresseurs", dans P. LALONDE, J. AUBUT, F. GRUNBERG et al. (dir.), Psychiatrie clinique: une approche bio-psycho-sociale, Boucherville, Gaëtan Morin.

MESSER, S.B. (1998). "Les croyances des psychothérapeutes intégratifs et éclectiques: un examen critique», dans J.C. NORCROSS et M.R. GOLDFRIED (dir.), Psychothérapie intégrative, Paris, Desclée de Brouwer.

ROYCE, J.R. et L.P. MOS (1980). Manual: Psycho-epistemological Profile, Center for advanced study in theoretical psychology, Edmonton, Université de l'Alberta.

RYLE, A. (1990). Cognitive Analytic Therapy: Active Participation in Change, Chichester, Wiley.

WEISSMAN, M.M., J.C. MARKOWITZ et G.L. KLERMAN (2006 [2000]). Guide de psychothérapie interpersonnelle, traduction française, New York, Basic Books. 